Voix et Images

voixetimages

\title{
Jean Fisette, le Texte automatiste, Essai de théorie/pratique de sémiotique textuelle
}

\section{Pierre Hébert}

Volume 4, numéro 3, avril 1979

Louis-Philippe Hébert

URI : https://id.erudit.org/iderudit/200183ar

DOI : https://doi.org/10.7202/200183ar

Aller au sommaire du numéro

Éditeur(s)

Les Presses de l'Université du Québec

ISSN

0318-9201 (imprimé)

1705-933X (numérique)

Découvrir la revue

Citer ce compte rendu

Hébert, P. (1979). Compte rendu de [Jean Fisette, le Texte automatiste, Essai de théorie/pratique de sémiotique textuelle]. Voix et Images, 4(3), 551-553.

https://doi.org/10.7202/200183ar d'utilisation que vous pouvez consulter en ligne.

https://apropos.erudit.org/fr/usagers/politique-dutilisation/ 


\section{Jean Fisette}

\section{le Texte automatiste, Essai de théorie/pratique} de sémiotique textuelle.

Certains ouvrages doivent être lus. On me permettra de situer dans cette catégorie le travail de Jean Fisette.

Le Texte automatiste satisfera l'appétit du théoricien de la littérature par la pertinence des modèles appliqués aux œuvres et il éclairera le "simple" (adjectif euphorique) lecteur du Vierge incendié (Paul-Marie Lapointe), du Refus global (Borduas) et d'Yeux fixes (Roland Giguère). Ainsi cette étude équilibre-t-elle la création théorique et l'enracinement critique. 
Constituant le corpus, les trois cuvres mentionnées sont groupées en vertu d'une perspective historique : elles marquent "l'éclosion d'un nouvel esprit au Québec "entre 1945 et 1951, la naissance de l'écriture automatiste. Le doigt entre l'arbre et l'écorce, le critique convoite d'abord la description des œuvres pour elles-mêmes, créant pour chacune un modèle approprié : “chacune des cuvres commande une méthode d'analyse particulière qui ne sera pas apportée artificiellement comme un a priori » (p. 17); puis, à un niveau supérieur, méta-critique, apparaîtra le Texte automatiste, carrefour des structures des ceuvres individuelles, des systèmes rhétoriques. Et quel langage choisir? «Notre travail s'inscrit dans un courant de recherche actuel qui consiste à dégager la signification d'un texte, non à partir de ce qu'il veut dire [...] mais de ce qu'il nous semble être comme construction de mots et de ce que sa substance (structure linguistique) peut signifier» (p. 21). L'opportunité du langage et de l'appareil linguistique ne fait dès lors aucun doute.

L'analyse du Vierge incendié occupe une place de choix, tant par l'espace que par la qualité, et, à l'instar des autres études, repose «sur le postulat de l'œuvre considérée comme "système clos" (p. 14). Le Vierge incendié, affirme Jean Fisette, n'est pas «illisible", comme le pensent la plupart des lecteurs critiques, mais trop lisible: “l'impression générale d' "illisibilité" semble provenir plutôt de la trop grande diversité de ces "prises» de lecture, portes d'entrée - et de sortie - au texte» (p. 27).

Pour lire le Vierge incendié, Jean Fisette met à contribution les distinctions de Hjelmslev et ouvre trois voies vers l'œuvre : le contenu (substance et forme), proposant une “sélection sémique», l'expression (substance et forme), où l'enjeu consiste à démont(r)er les mécanismes de production du sens (l'examen des particules, par exemple), et la forme (expression et contenu), qui présente quatre types isotopiques soumis à un examen minutieux et convaincant. "Au terme de l'analyse, nous découvrons que dans la réalité textuelle, à la différence des dénominations linguistiques que nous avions adoptées, la particule DE ainsi que les rapports bidirectionnels entre les isotopies ne remplissent pas une fonction de détermination, mais bien d'indétermination, appel à tous les possibles" ( $p$. 75). L'œuvre est ouverte...

L'approche du Refus global procède d'une manière différente: “Nous retenons une série de fragments du texte et nous en déduisons un système; ce système, nous l'utilisons ensuite comme grille pour lire l'ensemble du texte» (p. 77). L'hypothèse étayée par le critique s'offre ainsi : "Le Refus global est essentiellement le produit d'un système lexical fondé sur la figure d'opposition et qui se prête à divers types de combinaison» ( $p$. 78). Ce système lexical s'établit en deux paradigmes, euphorique (valeur axiologique positive) et dysphorique (valeur négative). "Une catégorie de formules syntaxiques et de termes agissent comme opérateurs qui permettent, 
stylistiquement, la réalisation de deux types d'énoncés, soit des formules de stéréotypes (combinaison horizontale, agençant des valeurs + et - ), soit des concaténations de lexèmes (combinaisons verticales, agençant des lexèmes de même valeur, + ou -)" (p. 136). Les " flottements du texte " se réalisent cependant autour des champs sémantiques «Histoire " et «Nous ".

Le malaise que crée la lecture d'Yeux fixes réside dans le caractère hybride de l'œuvre, à la fois récit et poésie. Ce constat commande l'approche, fondée sur un modèle narratif et un modèle linguïstique. Sur le plan narratif, la structure "d'Yeux fixes pourrait se comparer à une chaîne où les microstructures - maillons - sont à la fois indépendantes par leur cohésion interne et sont aussi fortement reliées entre elles" ( $p$. 114). La structure linguistique entraîne la mise en relief de deux niveaux de fiction, l' "épistémologie" et le "narratif", ces deux niveaux ouvrant deux voies dans la lecture d'un même fragment de texte, "paradigmatique et syntagmatique, le premier correspondant au «Je» de la fiction épistémologique et le second au "Moi » de la fiction narrative» (p. 145).

Les trois analyses remontent finalement à leur source, le Texte automatiste. La diversité des structures rhétoriques mises à jour s'unifie dans le modèle actantiel de Greimas. Ainsi Jean Fisette démontre-t-il que chaque structure privilégie un axe particulier: le Refus global met en épingle l'axe de la participation, le Vierge incendié, l'axe du désir, et Yeux fixes, l'axe de la communication. Le lecteur clôt le volume satisfait, l'homogénéité du corpus est établie : "chacune des ceuvres parle des deux autres » (p. 147) à un niveau hiérarchiquement supérieur, un super-signe en quelque sorte, le Texte automatiste.

C'est peut-être le point de départ des trois études qui étonne davantage. Dans chaque cas, l'objet ou, plus précisément, une impression que crée l'objet, suscite ensuite le point de vue: l'impression d'illisibilité opposée à une "hyper-lisibilité" pour' le Vierge incendié, le choix de fragments et l'hypothèse des oppositions lexicales pour le Refus global, le malaise créé par la présence de la poésie et du récit dans Yeux fixes. Certes, l'auteur connaît cette «limite essentielle de la pratique critique", c'est-à-dire l'arbitraire des données initiales. Et la rentabilité critique de la démarche ne fait aucun doute. Mais que se serait-il produit si le(s) point(s) de départ avaient été autre(s)? Aurions-nous eu la même vision du Texte automatiste?

L'étude de Jean Fisette, très dense. et exigeante pour le lecteur, fait assurément avancer la théorie et la critique littéraires. Les rênes sont tenues avec assurance et jamais n'avons-nous l'impression que le texte est ligoté par un modèle. Au sortir de l'intervention critique, les œuvres respirent mieux. 


\section{ERRATUM}

La chronique intitulée "l'Écriture moderne. Encore. Enfin" qui a paru dans le volume IV, numéro 1, septembre 1978, traitait de Philippe Haeck, en particulier de Polyphonie, Montréal-Nord, VLB Éditeur, 1978. La rédaction vous prie de l'excuser de cette omission. 